\title{
Research on the Optical Computer Based on Photonic Chip
}

\author{
Lingling Zhao ${ }^{1, \text { a }}$, Xuemei Liü, b and Qiaoyun Sun ${ }^{3, \mathrm{c}}$ \\ ${ }^{1}$ Software Engineering, Beijing city University, Beijing, 100083, China \\ ${ }^{2}$ Software Engineering, Beijing city University, Beijing, 100083, China \\ ${ }^{3}$ Communication Engineering, Beijing city University, Beijing, 100083, China \\ azhaolingling@bcu.edu.cn, ${ }^{b} x u e m l i u @ 163 . c o m,{ }^{c}$ sunqy@bcu.edu.cn
}

Keywords: Optical computer; Electronic computer; Hardware

\begin{abstract}
Today's computer science and technology is developing rapidly with the development of optical technology. But any kind of technology development will be subject to its own limitations inherent physical properties. With the development of the VLSI, best integrated on a single chip electronic components are almost saturated. Contemporary computer has been developed to an unprecedented height; you can almost say that the development computer has reached a bottleneck. In this context, the photon computer technology emerged, although still immature, but the prospects are very bright.
\end{abstract}

\section{Introduction}

An optical computer is also called as a photonic computer, which is manufactured by using Nano plasma components as the core and computes information via optical signals. This computer for information processing with the light as the carrier is called as the optical computer.

Photonic computer is a kind of new computer that has high speed, big storage space and small information distortion, and low heat dissipation. So it is one of the important direction of computer research. For fast processing and analysis needs of large data systems in the defense, aerospace, large-scale weather forecasts and other fields, many countries have been working on supercomputers, such as our Tianhe II supercomputer, its running speed reached 33.86pflop, is the world's fastest supercomputers, but can be achieved by ordinary photons computer run faster than trillion, so the development of photonic computers is helpful to supercomputers. However, developing photonic computer is not so simple as computers, there are many technical problem difficult to overcome, for example-photonic chips.

Uncertainty and instability caused difficulties in photonic chip research and development. The U.S. computer chip giant Intel Corporation has announced that they and the University of California, Santa Barbara (UCSB) have successfully developed the world's first silicon manufacturing process using standard electric hybrid silicon laser. However, there is still a big gap between this chip and that really used in photonic computers.

However, compared to the difficulties in the development of photonic computer, its great advantage is more attractive, at present, many of the world's leading electronics or computer companies are committed to the development of photonic computers, I believe in the near future, photonic computer will go into people's life and become the mainstream of computers.

\section{Optical Computer Based on the Photonic Chip}

The first photonic computer is developed out at the American Bell Laboratory, which is made of gallium arsenide optic switches, can compute 1 billion times per second and process the information by using the optic pulse. The quantity and speed of the information processing based on optical pulse signals is far over it of a PC according to the physical theory. Although this optic computer has a gap with the theoretical optical computer, it has a bright future.

The photonic computer can perform the data calculation, data transfer and data storage by replacing the electronics with the photons. Different wavelengths represent different data in a 
photonic computer. After the photons replace electronics and optical calculation replaces the electronic calculation, the performance of a photonic computer is higher than it of an electronic computer based on binary calculation with 0 and 1 state. A photonic computer can quickly process the tasks with high complexity and heavy calculation workload in a concurrent manner. The calculation speed of a photonic computer is far over it of an electronic computer. The photonic computer has a fault tolerance performance like the brain. If one component is damaged or fails in the system, it will not affect final calculation results. The photon features low transmission deformation, low consumption and low heat dissipation in optical medium and lower requirement for the ambience compared to an electronic computer.

\section{Weaknesses of Electronic Computer}

An electronic computer can transform the electronic signals to logic signals. The generated pulse can transmit data via metal wires. First, any metal wire has a resistance and capacitance. From the electromagnetic knowledge, we can know that combination of resistance and capacitance will generate "resistance" to the electronics transmitted $\mathrm{n}$ a lead and reduce its transmission speed in the lead. Electronics will react to quick external change bluntly. When the carrier to transmit information has a higher frequency, namely the signals change quickly, the electric signals in the lead cannot keep abreast with change of information signals to transmit. Secondly, although the central processor of an electronic computer can quickly process data and the main memory can permit high throughput. All data signals should be transmitted via the bus. When the current density of the bus is too high, it will generate higher electromagnetic disturbance, so calculation speed of the electronic computers will be limited like the vehicle passing speed restricted due to narrow junction of an express road. In addition, the integration density of the electronic parts used in a computer is restricted due to disturbance of the quantum effect.

\section{Strengths of Photonic Computer}

An electronic computer has some weaknesses. The carrier, which transmits information with photons, has the following strengths:

High Volume Of Information To Transmit And Process. The optic parts permit to pass highfrequency light and wide-range light. When two beams interfere with each other, they should have same frequency, consistent vibration direction and constant initial phase difference. When one optical fiber can transmit many light waves with different wave lengths or with same wave length and different vibration directions in parallel, they will not interfere with each other.

High Speed Information Calculation And Small Deformation In Information Transmission. A photonic computer features stronger concurrent processing capability and higher calculation speed. An electronic computer will conduct via some mutually insulated leads. An optic computer has "no" leads. No resistance, capacitance, inductance and potential difference are available when the light is transmitted in optic medium, so the information deformation and deformation caused by transmission is very small. Switching speed of optic components is higher much than it of electronic components and its requirements for the operation environment is lower than them of an electronic computer.

Ultra-large Information Memory Capacity. Compared to an electronic computer, a photonic computer can store an ultra-large information. A photonic computer has a laser as a very ideal light emission source. Photons can transmit without a lead. Even photons intersect with each other, they will not directly affect each other. In fact, the parallel channels of a photonic computer for information transmission without a lead has limitless density.

Low Power Consumption And Low Heat Dissipation. Although the current is very low in an integrated circuit, the power consumption is still high due to increase of integration level. A super computer will consume more power. An optic computer is an energy-saving product. A laser source requires some energy, the light will consume few energy in transmission and switching. A miniature and portable photonic computer will be developed. 
Based on the strengths of an optic computer, it will be extensively applied in different fields, especially in some special fields, e.g. some complicated and variable processes such as weather forecasting. An optical computer can also be used for call transmission. It is a very important step for a computer to process data and information with optical waves instead of current. In future, an optical computer will bring stronger calculation capability and higher processing speed to us.

\section{Hardware of Optical Computer}

In future, optical signals are replaced with electronic signals in the whole architecture of an optical computer. After optical signals are triggered by an input device, they are transmitted to the CPU for processing and output. Its principle is like it of a current electronic computers. The optical computer will communicate based on the optical signals. The following part simply predicts each fittings of a photonic computer.

Optical Processor CPU. As the core of a photonic computer, it is still developed and studied as an independent product. The calculation speed can reach 8000 billion times/time and is 1000 time of it of a common digital signal processor.

Mainboard. The mainboard may be replaced with the optical signal generator or transmitter in an optical computer. In future, the mainboard is not important as the current mainboard because optical signals may be generated in a power. Optical signals can be transmitted simply via the connection between CPU and other objects, so the mainboard is not necessary.

Harddisk and Memory. The harddiskk of a photonic computer can complete the function of a memory and harddisk. The memory will disappear. Now the principle of a computer is changed. The processor can directly read, run and process data from the high-speed harddisk. A new optical memory is a liquid memory, which can penetrate light. The data will be directly recorded in each atom. The high-speed atoms can read data at a high speed to replace the memory.

Graphics card And Display. The graphics card will be integrated with a display. The current LCD represents an embryonic form of a display in future. In future, the display will become a liquid display without point distance. It is also the ultra-thin display like a current display. The display panel will include a material, which will give out light under excitation. The display will display a graph with each atom as the light emission unit and display without point distance.

\section{Prospect of Optical Computer}

From the development course of an optical calculation and optical computer, it is not feasible to completely electronic computers with optical computers. Domestic and foreign researchers in the optical computer think to combine optics with the electronics, make up weaknesses of an electronic computer by using strengths such as space concurrence, high frequency bandwidth, no highfrequency emission and crossing interference, high space flexible interconnection and highcapacity information storage of optics, and exert stronger programmability and controllability of electronics. The optical and electronics hybrid special parallel high-performance computer should be studied to solve actual problems, which can not be completed in electronics.

The ideal optical computers can be based on optical processing, optical interconnection and transmission, electronic control and photo-electricity hybrid input/output general digital photoelectricity hybrid computer, but it faces to huge challenges in development, e.g. optical channel switch and logic signal transmission problem, lack of high-quality parallel two-dimensional photoelectric device array, architecture and graphic symbol logic theory of an optical computer on exploration stage.

Different high-end computers are emerging in a high-speed developing computer era. Theories and practices such as superconductivity computer, Nano computer, optical computer, DNA computer and quantum computer have evolved much. A computer has utterly change fields such as communication, medical treatment, education, military industry and scientific research. With development of IOT and cloud computing and exploration of human being in the optical fields, the optical computers will surely drive development of the human society with its strengths. 


\section{References}

[1] Cong Liangchen. Discussion on development and future of optical computer. Baidu paper library, 2015.

[2] Jin Yi. Approach to optical computer. Journal of Shanghai University, 2011.

[3] Li Bo. Development conditions of photonic computers. Fujian PC, 2013

[4] Liu Wei. Discussion on current conditions and development of photonic computers. Science and Technology Innovation Herald, 2011.

[5] Zhou Jiagao. Wonderful photonic computer. Chinese Times, 2001.

[6] Yang Yanni, Liu Peng, Li Chuanjiang. Discussion on photonic computer, Science and Technology vision, Science and technology vision, 2016.

[7] Chen Weizong, He Zhifeng, Liu Jun. Optical computer---photonics progress IV. Physics Bulletin, 2011.

[8] Zhao Shunjie. Discussion on development trend of computer science and technology, PC Knowledge and Technology, 2016. 